\title{
PLURICANONICAL SYSTEMS ON IRREGULAR 3-FOLDS OF GENERAL TYPE
}

\author{
JUNGKAI A. CHEN AND CHRISTOPHER D. HACON
}

\begin{abstract}
In this paper we prove that if $X$ is an irregular 3-fold with $\chi\left(\omega_{X}\right)>0$, then $\left|m K_{X}\right|$ is birational for all $m \geq 5$.
\end{abstract}

\section{Introduction}

Given a non-singular variety of general type, by definition, the pluricanonical system $\left|m K_{X}\right|$ defines a birational map $\varphi_{m}$ to a projective space, for all sufficiently big integers $m$. It is a natural question to try and find a reasonable effective bound for such integers $m$. When $\operatorname{dim} X=1$, it is classically known that $\varphi_{m}$ is an embedding for $m \geq 3$. For surfaces, by a result of [Bo], $\varphi_{m}$ is birational for $m \geq 5$. Recently, following ideas of Tsuji (cf. [Ts1], [HM], [Ta]), it has been shown that for any positive integer $n>0$, there exists a positive integer $r_{n}>0$ such that $\varphi_{m}$ is birational for $m \geq r_{n}$ for all varieties of general type and of dimension $n$. Tsuji has also shown [Ts2], that $r_{3} \leq 18\left(2^{9} \cdot 3^{7}\right)$ !. Recently Todorov [To], has shown that for all but finitely many families of 3-folds of general type, the 5-th canonical map is birational.

The purpose of this note is to study pluricanonical systems on irregular varieties, i.e. varieties with $q(X):=h^{0}\left(X, \Omega_{X}\right)>0$. In view of recent results on irregular varieties, one might hope that their geometry behaves similarly to that of surfaces. In this article we are in fact able to prove the following:

Theorem 1.1. Let $X$ be an irregular smooth projective threefold of general type with $\chi\left(\omega_{X}\right)>0$. Then $\left|m K_{X}+P\right|$ is birational for $m \geq 5$ and for all $P \in \operatorname{Pic}^{0}(X)$.

It is easy to see that this result is optimal. Consider in fact a surface $S$ of general type such that $\left|4 K_{S}\right|$ is not birational and a curve $C$ of general type. For all $P \in \operatorname{Pic}^{0}(C \times S) \cong \operatorname{Pic}^{0}(C)$ one sees that $\left|4 K_{C \times S}+P\right|$ is not birational.

The main technical ingredient is the technique developed in [CH1], where the Fourier-Mukai transform and vanishing theorems are used to study adjoint linear series on irregular varieties. For the reader's convenience, in Section 2 we recall the main result from [CH1]. Its

The first author was partially supported by NSC and NCTS of Taiwan.

The second author was partially supported by NSF research grant no: 0456363. 
application to pluricanonical system is summarized in Theorem 2.8. One finds a very sharp bound in Proposition 2.9 when the Albanese fiber dimension is small. Starting in Section 3, we will work on irregular varieties over curves. The case when $X$ maps to a curve with genus $g \geq 2$ is handled in Section 3 by a relatively straightforward application of the weak positivity of the relative dualizing sheaves. The case when $X$ maps to an elliptic curve is more subtle. We are unable to recover a general result in arbitrary dimension. However, by a careful study of the positivity of vector bundles over elliptic curves in Section 4, we are able to prove our main theorem for threefolds in Section 5.

Acknowledgments. We are indebted to Meng Chen, De-Qi Zhang, Yujiro Kawamata, I-Hsun Tsai, Hélène Esnault, Eckart Viehweg and Chin-Lung Wang for useful conversations and comments on this subject.

1.1. Notation. We work over the field of complex numbers $\mathbb{C}$. A $\mathbb{Q}$ Cartier divisor $D$ on a normal variety $X$ is nef if $D \cdot C \geq 0$ for any curve $C \subset X$. We say that two $\mathbb{Q}$-divisors $D_{1}, D_{2}$ are $\mathbb{Q}$-linearly equivalent $\left(D_{1} \sim_{\mathbb{Q}} D_{2}\right)$ if there exists an integer $m>0$ such that $m D_{i}$ are linearly equivalent. Numerical equivalence is denoted $\equiv$. And we write $D_{1} \geq D_{2}$ if $D_{1}-D_{2}$ is effective. For any projective variety $X, \operatorname{Pic}^{0}(X)$ denotes the abelian variety parametrizing the topologically trivial line bundles on $X$. If $A$ is an abelian variety, then we can identify $\hat{A}$ with $\operatorname{Pic}^{0}(A)$. We will denote by $\mathcal{P}$, the Poincaré line bundle on $A \times \hat{A}$.

\section{Linear series on irregular varieties}

We begin by recalling several important consequences of the theory of Fourier-Mukai transforms that will be needed throughout the paper.

Let $A$ be an abelian variety. Recall that there is a functor $\hat{\mathcal{S}}$ from the category of $\mathcal{O}_{A}$-modules to the category of $\mathcal{O}_{\hat{A}}$-modules defined by

$$
\hat{\mathcal{S}}(M)=\left(p_{\hat{A}}\right)_{*}\left(\mathcal{P} \otimes p_{A}^{*} M\right) .
$$

Here $p_{A}$ and $p_{\hat{A}}$ denote the projections of $A \times \hat{A}$ to $A$ and $\hat{A}$. Similarly we define $\mathcal{S}(N)=\left(p_{A}\right)_{*}\left(\mathcal{P} \otimes p_{\hat{A}^{*}}^{*}\right)$. Let $R \mathcal{S}$ (resp. $R \hat{\mathcal{S}}$ ) be the derived functor of $\mathcal{S}$ (resp. $\hat{\mathcal{S}}$ ) between the corresponding derived categories. By a result of Mukai (cf. [Mu] Theorem 2.2), we have:

Theorem 2.1. There exist isomorphisms of functors

$$
R \hat{\mathcal{S}} \circ R \mathcal{S} \cong\left(-1_{\hat{A}}\right)^{*}[-g] \quad \text { and } \quad R \mathcal{S} \circ R \hat{\mathcal{S}} \cong\left(-1_{A}\right)^{*}[-g] .
$$

A coherent sheaf $\mathcal{F}$ on an abelian variety $A$ is said to be $I T^{0}$ if $H^{i}(A, \mathcal{F} \otimes P)=0$ for all $i>0$ and all $P \in \operatorname{Pic}^{0}(A)$.

Lemma 2.2. Let $\mathcal{F}$ be a coherent $I T^{0}$ sheaf on an abelian variety $A$. If $\mathcal{F} \neq 0$, then $H^{0}(A, \mathcal{F} \otimes P) \neq 0$ for all $P \in \operatorname{Pic}^{0}(A)$. 
Proof. Please see [CH1] Lemma 2.1.

Lemma 2.3. Let $\mathcal{F} \neq 0$ be an $I T^{0}$ coherent sheaf on an abelian variety $A$. Suppose that there is a non-zero map $\mathcal{F} \rightarrow \mathbb{C}(z)$. Then the induced map $H^{0}(\mathcal{F} \otimes P) \rightarrow H^{0}(\mathbb{C}(z))$ is non-zero for general $P \in \operatorname{Pic}^{0}(A)$.

Proof. Please see [CH1] Proposition 2.3.

Here are some useful consequences:

Corollary 2.4. Let $X$ be a smooth projective variety and $a: X \rightarrow Z=$ $a(X) \subset A$ a morphism to an abelian variety $A$. Let $F$ be a general fiber and $x$ a point on $F$. Let $L$ be a divisor on $X$ such that $L \equiv a^{*} H+\Delta$ for a nef and big $\mathbb{Q}$-divisor $H$ and $a \mathbb{Q}$-divisor $\Delta \geq 0$ so that $\Delta$ has simple normal crossings support and $\lfloor\Delta\rfloor=0$. Suppose furthermore that $a_{*} \mathcal{O}_{X}\left(K_{X}+L\right) \neq 0$. Then we have:

(1) If $x \notin B s\left|\left(K_{X}+L\right)\right|_{F} \mid$, then $x \notin B s\left|K_{X}+L+a^{*} P\right|$ for a general $P \in \operatorname{Pic}^{0}(A)$.

(2) Let $x_{1}, x_{2}$ be two points on different general fibers $F_{1}, F_{2}$ respectively. If $x_{i} \notin B s\left|\left(K_{X}+L\right)\right|_{F_{i}} \mid$ for $i=1,2$, and one of the points $x_{i}$, say $x_{1}$, does not belong to $B s\left|K_{X}+L+a^{*} P\right|$ for all $P \in \operatorname{Pic}^{0}(A)$, then $\left|K_{X}+L+a^{*} P\right|$ separates $x_{1}, x_{2}$ for a general $P \in \operatorname{Pic}^{0}(A)$.

(3) Let $x_{1}, x_{2}$ be two distinct points on a general fiber $F$. If $x_{i} \notin$ $B s\left|\left(K_{X}+L\right)\right|_{F} \mid$ for $i=1,2,\left|\left(K_{X}+L\right)\right|_{F} \mid$ separates $x_{1}, x_{2}$, and one of $x_{i}$, say $x_{1}, \notin B s\left|K_{X}+L+a^{*} P\right|$ for all $P \in \operatorname{Pic}^{0}(A)$, then $\left|K_{X}+L+a^{*} P\right|$ separates $x_{1}, x_{2}$ for a general $P \in \operatorname{Pic}^{0}(A)$.

Proof. Let $z \in Z$ be a general point and $F$ the fiber over $z$. By $z$ being general, we mean that

$$
a_{*} \mathcal{O}_{X}\left(K_{X}+L\right) \otimes \mathbb{C}(z) \cong H^{0}\left(F, \mathcal{O}_{F}\left(K_{X}+L\right)\right) .
$$

We first look at the short exact sequence, obtained by evaluated at $x$ :

$$
(*) \quad 0 \rightarrow \mathcal{O}_{X}\left(K_{X}+L\right) \otimes \mathcal{I}_{x} \rightarrow \mathcal{O}_{X}\left(K_{X}+L\right) \rightarrow \mathbb{C}(x) \rightarrow 0 .
$$

Pushing forward to $Z$, we get

$$
0 \rightarrow a_{*}\left(\mathcal{O}_{X}\left(K_{X}+L\right) \otimes \mathcal{I}_{x}\right) \rightarrow a_{*} \mathcal{O}_{X}\left(K_{X}+L\right) \rightarrow \mathbb{C}(z) \rightarrow \ldots
$$

Since $x \notin B s\left|\left(K_{X}+L\right)\right|_{F} \mid$ and

$$
a_{*} \mathcal{O}_{X}\left(K_{X}+L\right) \otimes \mathbb{C}(z) \cong H^{0}\left(F, \mathcal{O}_{F}\left(K_{X}+L\right)\right),
$$

one sees that the induced map

$$
a_{*} \mathcal{O}_{X}\left(K_{X}+L\right) \rightarrow a_{*} \mathcal{O}_{X}\left(K_{X}+L\right) \otimes \mathbb{C}(z) \rightarrow \mathbb{C}(z)
$$

is non-zero, whence surjective.

By Corollary 10.15 of [Ko3], $a_{*} \mathcal{O}_{X}\left(K_{X}+L\right)$ is $I T^{0}$. Since $a_{*} \mathcal{O}_{X}\left(K_{X}+\right.$ $L) \neq 0$ by assumption, applying Lemma 2.3 to the sheaf $a_{*} \mathcal{O}_{X}\left(K_{X}+L\right)$, we get (1). 
Before we move forward to the proof of the next two statements, we remark that the assumption that $x_{1} \notin B s\left|K_{X}+L+a^{*} P\right|$ for all $P$ implies that $a_{*}\left(\mathcal{O}_{X}\left(K_{X}+L\right) \otimes \mathcal{I}_{x_{1}}\right)$ is $I T^{0}$ (by tensoring $(*)$ with $a^{*} P$ and replacing $x$ by $\left.x_{1}\right)$.

To see (3), we begin by looking at the exact sequence

$$
0 \rightarrow a_{*}\left(\mathcal{O}_{X}\left(K_{X}+L\right) \otimes \mathcal{I}_{x_{1}, x_{2}}\right) \rightarrow a_{*}\left(\mathcal{O}_{X}\left(K_{X}+L\right) \otimes \mathcal{I}_{x_{1}}\right) \rightarrow \mathbb{C}(z) \rightarrow \ldots
$$

The last map factors as

$$
\begin{gathered}
a_{*}\left(\mathcal{O}_{X}\left(K_{X}+L\right) \otimes \mathcal{I}_{x_{1}}\right) \rightarrow a_{*}\left(\mathcal{O}_{X}\left(K_{X}+L\right) \otimes \mathcal{I}_{x_{1}}\right) \otimes \mathbb{C}(z) \\
\rightarrow a_{*} \mathcal{O}_{X}\left(K_{X}+L\right) \otimes \mathbb{C}(z) \rightarrow \mathbb{C}(z) .
\end{gathered}
$$

It is obtained by evaluating at $x_{2}$. The assumption that $\mid\left(K_{X}+\right.$ $L)\left.\right|_{F} \mid$ separates $x_{1}, x_{2}$ shows that this is surjective. Again, by applying Lemma 2.3 to the sheaf $a_{*}\left(\mathcal{O}_{X}\left(K_{X}+L\right) \otimes \mathcal{I}_{x_{1}}\right)$, we are done.

Finally we consider (2). Again we have (assuming now that $x_{2}$ lies over $z)$ :

$$
0 \rightarrow a_{*}\left(\mathcal{O}_{X}\left(K_{X}+L\right) \otimes \mathcal{I}_{x_{1}, x_{2}}\right) \rightarrow a_{*}\left(\mathcal{O}_{X}\left(K_{X}+L\right) \otimes \mathcal{I}_{x_{1}}\right) \rightarrow \mathbb{C}(z) \rightarrow \ldots
$$

But now we look at the map

$$
\begin{gathered}
a_{*}\left(\mathcal{O}_{X}\left(K_{X}+L\right) \otimes \mathcal{I}_{x_{1}}\right) \rightarrow a_{*}\left(\mathcal{O}_{X}\left(K_{X}+L\right) \otimes \mathcal{I}_{x_{1}}\right) \otimes \mathbb{C}(z) \\
\rightarrow a_{*} \mathcal{O}_{X}\left(K_{X}+L\right) \otimes \mathbb{C}(z) \rightarrow \mathbb{C}(z)
\end{gathered}
$$

which is obtained by evaluating at $x_{2}$. Since $a\left(x_{1}\right) \neq a\left(x_{2}\right)$, it follows that $a_{*}\left(\mathcal{O}_{X}\left(K_{X}+L\right) \otimes \mathcal{I}_{x_{1}}\right) \otimes \mathbb{C}(z) \cong a_{*} \mathcal{O}_{X}\left(K_{X}+L\right) \otimes \mathbb{C}(z)$ and hence the above map is surjective. By Lemma 2.3 we are done.

We now fix some notation and conventions. For any $P \in \operatorname{Pic}^{0}(X)$, we define $\mathfrak{B}_{m, P}$ to be the fixed component of $\left|m K_{X}+a^{*} P\right|$ and $\mathfrak{B}_{m}$ (resp. $\mathfrak{B}_{m, F}$ ) to be the fixed component of $\left|m K_{X}\right|$ (resp. $\left|m K_{F}\right|$ ). After replacing $X$ by an appropriate birational model, we may and we usually do assume that $\mathfrak{B}_{m, P}, \mathfrak{B}_{m, F}$ have simple normal crossing support.

We need the following:

Lemma 2.5. Let $g: X \rightarrow W$ be a morphism with a general fiber $F$. Suppose that $\kappa(W) \geq 0$ and $K_{X}$ is $W$-big, i.e. $s K_{X} \geq g^{*} L$ for some ample divisor $L$ and some integer $s \gg 0$. Suppose further that $P_{m}(F)>0$ for some $m \geq 2$. Then after replacing $X$ by an appropriate birational model if necessary, there exist positive integers $a, b$ and there is a normal crossing divisor $B \in\left|a b(m-1) K_{X}-g^{*} b H\right|$ such that

$$
\left.\left\lfloor\frac{B}{a b}\right\rfloor\right|_{F} \leq \mathfrak{B}_{m, F}, \quad\left\lfloor\frac{B}{a b}\right\rfloor \leq \mathfrak{B}_{m, P},
$$

for all $P \in \operatorname{Pic}^{0}(W)$. Here $H$ is a given nef and big divisor on $W$ and $a, b$ are sufficiently large integers depending on $H$ and $K_{X}$.

Proof. Please see [CH1], Lemma 5.1. 
Definition 2.6. Let $a: X \rightarrow A$ be a morphism to an abelian variety and $D$ a Cartier divisor on $X$. Let

$$
V^{0}(D):=\left\{P \in \operatorname{Pic}^{0}(A) \mid h^{0}\left(X, \mathcal{O}_{X}(D) \otimes a^{*} P\right) \neq 0\right\} .
$$

We say that $D$ is full if $V^{0}(D)=\operatorname{Pic}^{0}(A)$.

Note that if $a_{*} \mathcal{O}_{X}(D)$ is $I T^{0}$ and non-zero, then by Lemma 2.2, $D$ is full.

2.7. General setting. We can now work on a more general setting. Let $a: X \rightarrow Z=a(X) \subset A$ be a morphism to an Abelian variety $A$. Usually, we take the Albanese map of $X$. Let $\nu: W \rightarrow Z$ be a desingularization of the Stein factorization over $Z$. Replacing $X$ by an appropriate birational model, we may assume that there is a morphism $g: X \rightarrow W$ with connected fibres. We assume that $X$ is of general type. It follows that $K_{X}$ is $W$-big. Let $F$ be the general fiber of $g$. In the following discussion, we will assume that $P_{m}(F)>0$ for some $m \geq 2$. We then take $B_{m}=\frac{B}{a b}$ with $B \in\left|a b(m-1) K_{X}-g^{*} b H\right|$ as in Lemma 2.5 and

$$
L_{m}:=(m-1) K_{X}-\left\lfloor B_{m}\right\rfloor \equiv \frac{1}{a} g^{*} H+\left\{B_{m}\right\}
$$

where $B_{m}=\left\lfloor B_{m}\right\rfloor+\left\{B_{m}\right\}$. By [Ko3] Theorem 10.15, since $H$ is the pull back of a nef and big divisor on $Z$, one sees that

$$
H^{i}\left(A, a_{*} \mathcal{O}_{X}\left(K_{X}+L_{m}\right) \otimes P\right) \cong H^{i}\left(W, g_{*} \mathcal{O}_{X}\left(K_{X}+L_{m}\right) \otimes \nu^{*} P\right)=0
$$

for all $P \in \operatorname{Pic}^{0}(A)$ and all $i>0$. In particular, $a_{*} \mathcal{O}_{X}\left(K_{X}+L_{m}\right)$ is $I T^{0}$. Recall that by Lemma 2.5 , one has $\left.\left\lfloor B_{m}\right\rfloor\right|_{F} \leq \mathfrak{B}_{m, F}$. Thus, $H^{0}\left(F, \mathcal{O}_{F}\left(\left.\left(K_{X}+L_{m}\right)\right|_{F}\right)\right) \cong H^{0}\left(F, \mathcal{O}_{F}\left(m K_{F}\right)\right)$. In particular, $a_{*} \mathcal{O}_{X}\left(K_{X}+\right.$ $\left.L_{m}\right) \neq 0$. By Lemma $2.2 K_{X}+L_{m}$ is full and hence so is $m K_{X}$.

Assume that for some integers $m, n \geq 1, m K_{X}$ and $n K_{X}$ are full and consider the morphism

$$
\begin{aligned}
\left|m K_{X}+a^{*} P_{1}\right| & +\left|n K_{X}+a^{*} P_{2}\right| \rightarrow\left|(m+n) K_{X}+a^{*}\left(P_{1}+P_{2}\right)\right| \\
& \cong\left|K_{X}+L_{m+n}+a^{*}\left(P_{1}+P_{2}\right)\right|,
\end{aligned}
$$

for all $P_{1}, P_{2}$. If $x \in X$ is a general point, then for general $P \in \operatorname{Pic}^{0}(A)$, $x$ is neither a base point of $m K_{X}+a^{*} P$ nor a base point of $n K_{X}+a^{*} P$. Therefore $x$ is not a base point of $\left|(m+n) K_{X}+a^{*} P\right|$ for all $P \in \operatorname{Pic}^{0}(A)$. And it follows that $x$ is not a base point of $\left|K_{X}+L_{m+n}+a^{*} P\right|$ since $x$ is general.

By Corollary 2.4, we can now deduce the main result of this section.

Theorem 2.8. Let $X$ be a smooth projective variety of general type, $a: X \rightarrow Z=a(X) \subset A$ a non-trivial morphism to an abelian variety, $F$ a general fiber of a and $m, n, t$ positive integers. If $m K_{X}, n K_{X}, t K_{X}$ are full, then:

(1) $\left|(m+n) K_{X}+a^{*} P\right|$ separates two general points on two distinct general fibers for general $P \in \operatorname{Pic}^{0}(A)$. 
(2) $\left|(m+n+t) K_{X}+a^{*} P\right|$ separates two general points on two distinct general fibers for all $P \in \operatorname{Pic}^{0}(A)$.

(3) If $(m+n) K_{F}$ is birational, then $\left|(m+n) K_{X}+a^{*} P\right|$ separates two general points on a general fiber for a general $P \in \operatorname{Pic}^{0}(A)$.

(4) If $(m+n) K_{F}$ is birational, then $\left|(m+n+t) K_{X}+a^{*} P\right|$ separates two general points on a general fiber for all $P \in \operatorname{Pic}^{0}(A)$.

Proof. (1) follows from Corollary 2.4 (2) since for two general points $x_{1}$ and $x_{2}$, we have seen that $x_{i} \notin B s\left|K_{X}+L_{m+n}+a^{*} P\right|$ for any $P \in \operatorname{Pic}^{0}(A)$ and so $x_{i} \notin B s\left|\left(K_{X}+L_{m+n}\right)\right|_{F} \mid$.

(3) follows from Corollary $2.4(3)$ since we assumed that $\left|(m+n) K_{F}\right|$ is birational and so, since by Lemma 2.5 we have $\left.\left(B_{m+n}\right)\right|_{F} \leq \mathfrak{B}_{m+n, F}$, then $\left|\left(K_{X}+L_{m+n}\right)\right|_{F} \mid$ is also birational.

(2) and (4) now follow by considering the morphism

$\left|(m+n) K_{X}+a^{*} P_{1}\right|+\left|t K_{X}+a^{*} P_{2}\right| \rightarrow\left|(m+n+t) K_{X}+a^{*}\left(P_{1}+P_{2}\right)\right|$.

Since $\left|(m+n) K_{X}+a^{*} P_{1}\right|$ separates $x_{1}, x_{2}$ for general $P_{1} \in \operatorname{Pic}^{0}(A)$ and $\left|t K_{X}+a^{*} P_{2}\right|$ does not vanish along $x_{1}, x_{2}$ for general $P_{2} \in \operatorname{Pic}^{0}(A)$, then $\left|(m+n+t) K_{X}+a^{*} P\right|$ separates $x_{1}, x_{2}$ for all $P \in \operatorname{Pic}^{0}(A)$.

Let $F$ be an irreducible component of a general fibre of the Albanese map $a=$ alb $: X \rightarrow a(X)$. Turning to a more detailed discussion, we now distinguish varieties according to their Albanese fiber dimension, i.e. $f:=\operatorname{dim} X-\operatorname{dim} a(X)$. We would like to remark that an analogous result holds for arbitrary non-trivial morphism to an abelian variety.

Proposition 2.9. Let $X$ be a smooth projective variety of general type with $q(X)>0$.

(1) If $f=0$, then

(a) $\left|m K_{X}+P\right|$ is birational for a general (resp. all) $P \in$ $\operatorname{Pic}^{0}(X)$ for $m \geq 4$ (resp. $m \geq 6$ ).

(b) If moreover $K_{X}$ is full, then $\left|m K_{X}+P\right|$ is birational for a general (resp. all) $P \in \operatorname{Pic}^{0}(X)$ for $m \geq 2$ (resp. $m \geq 3$ ).

(2) If $f=1$, then

(a) $\left|m K_{X}+P\right|$ is birational for a general (resp. all) $P \in$ $\operatorname{Pic}^{0}(X)$ and for $m \geq 4$ (resp. $m \geq 6$ ).

(b) If moreover $K_{X}$ is full, then $\left|m K_{X}+P\right|$ is birational for a general (resp. all) $P \in \operatorname{Pic}^{0}(X)$ and for $m \geq 3$ (resp. $m \geq 4)$.

(3) If $f=2$, then

(a) $\left|m K_{X}+P\right|$ is birational for a general (resp. all) $P \in$ $\operatorname{Pic}^{0}(X)$ and for $m \geq 5$ (resp. $m \geq 7$ ).

(b) If moreover $K_{X}$ is full, then $\left|m K_{X}+P\right|$ is birational for a general (resp. all) $P \in \operatorname{Pic}^{0}(X)$ and for $m \geq 5$ (resp. $m \geq 6$ ). 
Proof. If $f=0$, then $X$ is generically finite over its Albanese variety. By Lemma $2.2 K_{X}+L_{2}$ is full and hence so is $2 K_{X}$. Clearly $\left|m K_{F}\right|$ separates points on fibers for $m \geq 1$, so $(a)$ and $(b)$ follow from Theorem 2.8 , letting $m=n=t=2$. If we assume that $K_{X}$ is full, then we let $m=n=t=1$.

If $f \geq 1$, one sees that for $m \geq 2, m K_{X}$ is full if and only if $P_{m}(F) \neq$ 0 . Since $F$ is of general type, for $f \leq 2$ it is well known that $P_{m}(F)>0$ for all $m \geq 2$. Therefore $m K_{X}$ is full for $m \geq 2$.

If $f=1, m K_{F}$ is birational for $m \geq 3$. By Theorem 2.8, we have $(a)$ and $(b)$, by taking $m=n=t=2$. If we assume that $K_{X}$ is full, then let $m=2, n=t=1$.

Finally, if $f=2$, then $m K_{F}$ is birational for $m \geq 5$. By Theorem 2.8 , we have $(a)$ and $(b)$ by letting $m=3, n=t=2$. If we assume that $K_{X}$ is full, then we let $m=4, n=t=1$.

\section{Varieties over curves of genus at least 2}

In this section, we study the case in which the Albanese image is a curve of $g \geq 2$.

Proposition 3.1. Let $a: X \rightarrow C$ be a morphism from a smooth projective variety of general type to a curve of genus $g(C) \geq 2$ with a (connected) general fiber $F$. Suppose that for some $m \geq 4,\left|m K_{F}\right|$ is birational. Then $\left|m K_{X}+a^{*} P\right|$ is birational for all $P \in \operatorname{Pic}^{0}(C)$.

Proof. Fix an integer $k \gg 0$ such that $P_{2 k}(F)>0$. We write

$$
2 k K_{X}-3 k F=2 k K_{X / C}+2 k a^{*} K_{C}-3 k F=2 k K_{X / C}+a^{*} H,
$$

for some ample line bundle $H$ of degree $(4 g(C)-7) k$. By the weak positivity of $a_{*}\left(\omega_{X / C}^{2 k}\right)$ (cf. [V1]), one has that the restriction map

$$
H^{0}\left(X, \mathcal{O}_{X}\left(2 n K_{X}-3 n F\right)\right) \rightarrow H^{0}\left(F, \mathcal{O}_{F}\left(2 n K_{F}\right)\right),
$$

is surjective for all $n \gg k$ sufficiently large and divisible by $k$ and by $m$. Therefore, $a_{*}\left(\omega_{X}^{2 n}(-3 n F)\right)$ is a vector bundle of rank $P_{2 n}(F)$ and degree at least $P_{2 n}(F)(4 g(C)-7) n$. Since $F$ is of general type, by RiemannRoch, one sees that $2 K_{X}-3 F$ is big. We write $\left|2 n K_{X}-3 n F\right|=$ $\left|N_{n}\right|+B_{n}$ where $B_{n}$ is the fixed part. We have $2 n K_{X} \sim M_{n}+B_{n}=$ $\left(N_{n}+3 n F\right)+B_{n}$. After replacing $X$ by an appropriate birational model, we may assume that $\left|N_{n}\right|,\left|M_{n}\right|$ are free and that $B_{n}$ is a divisor with normal crossing support. Note that $M_{n}$ is nef and big. We write $\left|2 n K_{F}\right|=\left|\left(M_{n}\right)\right|_{F} \mid+\mathfrak{B}_{2 n, F}$. By the above surjection, we have that $\mathfrak{B}_{2 n, F}=\left.\left(B_{n}\right)\right|_{F}$. Let

We have $(m-3) K_{X} \equiv \frac{(m-3) M_{n}}{2 n}+\frac{(m-3) B_{n}}{2 n}$ and $2 K_{X}-3 F \equiv \frac{N_{n}}{n}+\frac{B_{n}}{n}$.

$$
L_{m}:=(m-1) K_{X}-\left\lfloor\frac{(m-1) B_{n}}{2 n}\right\rfloor .
$$


Since $m$ divides $n$, we have that $B_{n} \leq \frac{n}{m} B_{m}$. Therefore,

$$
K_{X}+L_{m} \geq m K_{X}-\left\lfloor\frac{m-1}{2 n} \frac{n}{m} B_{m}\right\rfloor \geq m K_{X}-\left\lfloor\frac{1}{2} B_{m}\right\rfloor .
$$

Similarly,

$$
K_{F}+\left.L_{m}\right|_{F} \geq m K_{F}-\left\lfloor\frac{m-1}{2 n} \mathfrak{B}_{2 n, F}\right\rfloor \geq m K_{F}-\left\lfloor\frac{1}{2} \mathfrak{B}_{2 m, F}\right\rfloor,
$$

and $\left|K_{F}+L_{m}\right|_{F}|\cong| m K_{F} \mid \neq \emptyset$. Note that

$$
L_{m} \equiv \frac{(m-3) M_{n}}{2 n}+\frac{N_{n}}{n}+\left\{\frac{(m-1) B_{n}}{2 n}\right\}+3 F .
$$

By Kawamata-Viehweg vanishing, one has that

$$
H^{1}\left(X, \mathcal{O}_{X}\left(K_{X}+L_{m}+a^{*} P-s F\right)\right)=0 \quad \text { for } s \leq 3, P \in \operatorname{Pic}^{0}(C) \text {. }
$$

Consider the short exact sequence:

$$
\begin{gathered}
0 \rightarrow \mathcal{O}_{X}\left(K_{X}+L_{m}+a^{*} P-F_{1}-F_{2}\right) \rightarrow \mathcal{O}_{X}\left(K_{X}+L_{m}+a^{*} P\right) \\
\rightarrow \mathcal{O}_{F_{1}}\left(K_{F_{1}}+\left.L_{m}\right|_{F_{1}}\right) \oplus \mathcal{O}_{F_{2}}\left(K_{F_{2}}+\left.L_{m}\right|_{F_{2}}\right) \rightarrow 0 .
\end{gathered}
$$

One sees that $\left|K_{X}+L_{m}+a^{*} P\right|$ separates general points on distinct general fibers $F_{1}$ and $F_{2}$.

Similarly, one has the surjection

$$
H^{0}\left(X, \mathcal{O}_{X}\left(K_{X}+L_{m}+a^{*} P\right)\right) \rightarrow H^{0}\left(F, \mathcal{O}_{F}\left(K_{F}+L_{m}\right)\right) .
$$

If $\left|m K_{F}\right|$ is birational then $\left|K_{X}+L_{m}+a^{*} P\right|$ separates general points on $F$ and so does $\left|m K_{X}+a^{*} P\right|$. This completes the proof.

\section{Varieties over elliptic curve}

In this section, we consider varieties $X$ of general type admitting a morphism to an elliptic curve $a: X \rightarrow C$. We will use the results and the notation of $[\mathrm{At}]$.

Lemma 4.1. For any integer $m \geq 2$, let $\mathcal{I}:=\mathcal{O}_{X}\left(-\left\lfloor B_{m}\right\rfloor\right)$ be as in (2.7). Then $a_{*}\left(\omega_{X}^{m}\right)=a_{*}\left(\omega_{X}^{m} \otimes \mathcal{I}\right)$ is an IT vector bundle of rank $P_{m}(F)$.

Proof. Clearly, we have an inclusion $a_{*}\left(\omega_{X}^{m} \otimes \mathcal{I}\right) \hookrightarrow a_{*}\left(\omega_{X}^{m}\right)$. As in (2.7), one sees that they have the same rank. Let $\mathcal{Q}$ be the cokernel. Then $\mathcal{Q}$ has generic rank 0 and hence is supported on finitely many points. If $\mathcal{Q} \neq 0$, then $h^{0}(C, \mathcal{Q}) \neq 0$. However, $a_{*}\left(\omega_{X}^{m} \otimes \mathcal{I}\right)$ is $I T^{0}$ and $h^{0}\left(a_{*}\left(\omega_{X}^{m} \otimes \mathcal{I}\right)\right)=h^{0}\left(a_{*}\left(\omega_{X}^{m}\right)\right)$. This is a contradiction. Therefore, $\mathcal{Q}=0$.

For simplicity, we will write $E_{m}:=a_{*}\left(\omega_{X}^{m}\right)$.

Definition 4.2. Let $E$ be a vector bundle on an elliptic curve $C$. We define

$$
d(E):=\max \{\operatorname{deg}(L) \mid L \subset E \text { is a rank one subbundle }\} .
$$


Recall that a maximal splitting of a vector bundle $E$ of rank $r$ (on an elliptic curve $C)$ is a sequence of vector bundles $\left(L_{1}, L_{2}, \ldots, L_{r}\right)$ such that $L_{1}$ is a line bundle of maximum degree contained in $E$ and $\left(L_{2}, \ldots, L_{r}\right)$ is a maximal splitting of $E / L_{1}$. Therefore $d(E)=\operatorname{deg}\left(L_{1}\right)$. Recall also that by a result of Atiyah [At], given an indecomposable vector bundle $E$ of rank $r$ on an elliptic curve, there is a maximal splitting $\left(L_{1}, L_{2}, \ldots, L_{r}\right)$ such that $L_{1} \leq L_{2} \leq \ldots \leq L_{r}$ are line bundles, where $L_{i} \leq L_{i+1}$ denotes that there is an injection $L_{i} \hookrightarrow L_{i+1}$. Moreover, if $E$ is a vector bundle of rank $r$ and degree $0 \leq s \leq r$, then $E$ has a maximal splitting with $L_{1}=\ldots=L_{r-s}=\mathcal{O}$, and $L_{r-s+1}=\ldots=L_{r}=L$ for some line bundle $L$ of degree 1 . Notice that

$$
\lfloor\mu(E)\rfloor=d(E)=\operatorname{deg}\left(L_{1}\right) .
$$

We also remark, that by a result of $\mathrm{Tu}[\mathrm{Tu}]$, any indecomposable vector bundle is semistable.

Lemma 4.3. Let $E$ be an indecomposable vector bundle on an elliptic curve, then the following are equivalent

(1) $\operatorname{deg}(E)>0$,

(2) $E$ is $I T^{0}$,

(3) $E$ is ample.

Proof. (1) $\Rightarrow$ (2). If $\operatorname{deg}(E)>0$ and $h^{1}(E \otimes P) \neq 0$ for some $P \in \operatorname{Pic}^{0}(E)$, then $h^{0}\left(E^{*} \otimes P^{*}\right) \neq 0$ and hence there is a non-zero homomorphism $P \rightarrow E^{*}$. This is impossible as by $[\mathrm{Tu}], E^{*}$ is semistable of negative slope.

$(2) \Rightarrow(3)$. Let $E$ be an $I T^{0}$ vector bundle. By a result of Hartshorne (cf. [Ha] Theorem 1.3), it suffices to show that every quotient line bundle has degree $\geq 1$. Let $L$ be a quotient line bundle of $E$. It is easy to see that $E$ being $I T^{0}$ implies that $L$ is $I T^{0}$ and hence has degree $\geq 1$.

$(3) \Rightarrow(1)$. If $E$ is ample, then $\operatorname{det}(E)$ is an ample line bundle. So $\operatorname{deg}(E)=\operatorname{deg}(\operatorname{det}(E))>0$.

Lemma 4.4. Let $E$ be an indecomposable vector bundle on an elliptic curve with $d(E) \geq 1$. Then $E$ is generically generated by its global sections.

Proof. This can be easily proven by induction on rank. If the rank of $E$ is 1 , then the statement follows from the fact that $h^{0}(E)>0$. Suppose that the statement is true when the rank is $<r$. Given $E$ of rank $r$, the quotient bundle $F=E / L_{1}$ has a decomposition $F=\oplus F_{i}$ where each $F_{i}$ is indecomposable with $d\left(F_{i}\right) \geq 1$. It follows from the inductive hypothesis that each $F_{i}$ and hence also $F$ is generically generated by its global sections. Since $H^{1}\left(L_{1}\right)=0$, it follows that $0 \rightarrow H^{0}\left(L_{1}\right) \rightarrow$ $H^{0}(E) \rightarrow H^{0}(F) \rightarrow 0$ is exact. Therefore we have a commutative 
diagram

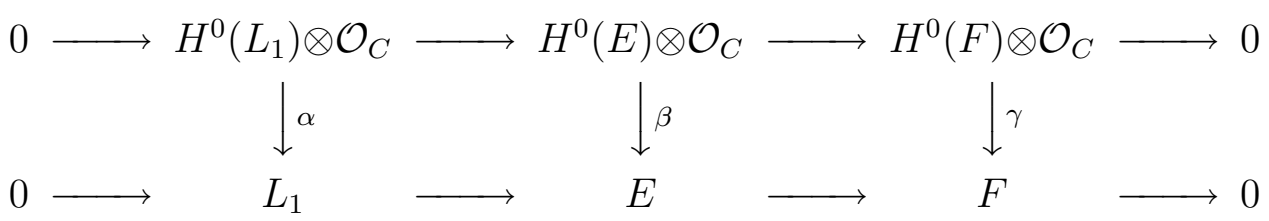

Since $\alpha$ and $\gamma$ are surjective on an open set then so is $\beta$.

Definition 4.5. For any vector bundle $E$, we write $E=\oplus E_{i}$ for its decomposition in to indecomposable vector bundles. We define $\nu(E):=$ $\min \mu\left(E_{i}\right)$.

Theorem 4.6. Let $a: X \rightarrow C$ be a morphism from a smooth projective variety of general type to an elliptic curve, $F$ a general fiber and $P \in$ $\operatorname{Pic}^{0}(C)$. If $\left|m K_{F}\right|$ is birational for some $m \geq 2$ and $\nu\left(a_{*} \omega_{X}^{m}\right) \geq 1$, then $\left|m K_{X}+a^{*} P\right|$ separates points on $F$.

Proof. This follows easily from the above Lemma 4.4 .

Lemma 4.7. Let $E, F$ be $I T^{0}$ vector bundles, then $E \otimes F$ is $I T^{0}$.

Proof. By Lemma 4.3, one sees that for a vector bundle, being $I T^{0}$ is equivalent to being ample. Moreover, if $E, F$ are ample, then $E \otimes F$ is ample. The Lemma now follows easily.

Lemma 4.8. Let $E_{1}, E_{2}$ be indecomposable vector bundles. Then Hom $\left(E_{1}, E_{2}\right) \neq 0$ implies that $\mu\left(E_{2}\right) \geq \mu\left(E_{1}\right)$.

Proof. See $[\mathrm{Tu}]$.

Lemma 4.9. Let $E$ be an $I T^{0}$ vector bundle which admits a short exact sequence

$$
0 \rightarrow F \rightarrow E \rightarrow Q \rightarrow 0
$$

such that $Q$ has generic rank $\leq 1$. Then $\nu(E) \geq \min \{1, \nu(F)\}$.

Proof. We distinguish the follows cases:

1. $Q$ is torsion.

For every indecomposable component of $E$, say $E_{i}$, there exists a nonzero map from an indecomposable component $F_{j}$ of $F$. By Lemma 4.8, $\mu\left(E_{i}\right) \geq \mu\left(F_{j}\right)$. So $\nu(E) \geq \nu(F)$. Notice that this step did not require $E$ to be $I T^{0}$.

2. $Q$ is torsion-free.

Then $Q$ is a line bundle. Since $E$ is $I T^{0}$, it follows that $Q$ is $I T^{0}$, hence $\mu(Q) \geq 1$. For any $E_{i}$, there exists a non-zero map from a component $F_{j}$ of $F$ unless $E_{i} \cong Q$. In either case, we have $\mu\left(E_{i}\right) \geq \min \{1, \nu(F)\}$.

3. In general, let $L:=Q^{* *}$, and $K:=\operatorname{ker}(E \rightarrow L)$. Then it is clear that $K$ fits into a short exact sequence

$$
0 \rightarrow F \rightarrow K \rightarrow Q_{\text {tor }} \rightarrow 0 .
$$

Thus $\nu(K) \geq \nu(F)$ by (1). By $(2), \nu(E) \geq \min \{1, \nu(F)\}$. 


\section{Proof of the main Theorem}

Proof of Theorem 1.1. We distinguish the follows cases according to their Albanese fiber dimension:

1. $f:=\operatorname{dim} X-\operatorname{dim} \operatorname{alb}(X)=0$.

It follows from generic vanishing theorem (cf. [GL1], [GL2]) that if $\chi\left(\omega_{X}\right)>0$, then $K_{X}$ is full. Therefore, by Proposition 2.9, we are done.

2. $f=1$.

By [Ko2] Corollary 3.2, $\chi\left(\omega_{X}\right)=\chi\left(a_{*} \omega_{X}\right)-\chi\left(R^{1} a_{*} \omega_{X}\right)$ and by [Ko1] Proposition 7.6, $R^{1} a_{*} \omega_{X}=\omega_{W}$. Since $W$ is of maximal Albanese dimension, by Theorem 1 of [GL1], $\chi\left(\omega_{W}\right) \geq 0$. Hence $\chi\left(a_{*} \omega_{X}\right) \geq$ $\chi\left(\omega_{X}\right)>0$. By the generalized generic vanishing theorem of [CH2], Proposition 2.2, one sees that $a_{*} \omega_{X}$ is full and hence so is $K_{X}$. Therefore, by Proposition 2.9, we are done with this case.

3. $f=2$.

Let $a: X \rightarrow C$ be the Albanese map. Note that $a$ has connected fibers. Consider in its Stein factorization $X \rightarrow W \rightarrow C$. Since $W$ is normal, it is a smooth curve. There are induced maps of abelian varieties $\operatorname{Alb}(X) \rightarrow \operatorname{Alb}(W) \rightarrow \operatorname{Alb}(C)$ which are easily seen to be isomorphisms, so that $W=C$.

3.a. If $g(C) \geq 2$.

This case follows from Proposition 3.1.

3.b. $g(C)=1$.

We may assume that $q(X)=1$ and thus $p_{g}(X) \geq 1$. We have that

$$
\chi\left(X, \omega_{X}\right)=\chi\left(C, a_{*} \omega_{X}\right)-\chi\left(C, R^{1} a_{*} \omega_{X}\right)+\chi\left(C, R^{2} a_{*} \omega_{X}\right) .
$$

Moreover, by [Ko1] Proposition 7.6, $R^{2} a_{*} \omega_{X}=\omega_{C}=\mathcal{O}_{C}$ and so $\chi\left(R^{2} a_{*} \omega_{X}\right)=0$. Now, $h^{1}\left(C, R^{1} a_{*} \omega_{X}\right)=q(X)-h^{0}\left(C, R^{2} a_{*} \omega_{X}\right)=0$ implies that $\chi\left(C, R^{1} a_{*} \omega_{X}\right) \geq 0$. Hence, one has $\chi\left(C, a_{*} \omega_{X}\right)>0$. So by the generalized generic vanishing of [CH2], Proposition 2.2, we see that $K_{X}$ is full as in Case 2.

Let $F$ be the general fiber of $a$ and $F_{0}$ be its minimal model. If $\left|4 K_{F}\right|$ is birational, then we are done by Theorem 2.8. So by Bombieri's classification [Bo], it remains to consider the case that $\left(K_{F_{0}}^{2}, p_{g}\left(F_{0}\right)\right)=$ $(1,2)$. We recall that for this surface, $q=0,\left|K_{F_{0}}\right|$ has a unique base point and $\left|2 K_{F_{0}}\right|$ is base point free.

Claim. Let $E_{m}=a_{*}\left(\omega_{X}^{m}\right)$. Then $\nu\left(E_{m}\right) \geq 1$ for $m \geq 5$.

Grant this for the time being. Then, by Lemma 4.4, for any $P \in$ $\operatorname{Pic}^{0}(C) \cong \operatorname{Pic}^{0}(X)$, every indecomposable component of $E_{m} \otimes P$ for $m \geq 5$ is generically generated by global sections. That is, there exists an open set $U \subset C$ and a surjection

$$
\left.H^{0}\left(E_{m} \otimes P\right) \otimes \mathcal{O}_{U} \rightarrow\left(E_{m} \otimes P\right)\right|_{\mathcal{O}_{U}} .
$$

In particular, there is a surjection

$$
H^{0}\left(X, \omega_{X}^{m} \otimes a^{*} P\right) \rightarrow H^{0}\left(E_{m} \otimes P\right) \otimes \mathcal{O}_{U} \rightarrow E_{m} \otimes P \otimes k(y) \cong H^{0}\left(\omega_{F_{y}}^{m}\right),
$$


for general $y \in U$. If follows that $\left|m K_{X}+P\right|$ separates points on a general fiber $F_{y}$ for $m \geq 5$. Since $\left|m K_{X}+P\right|$ also separates points on different general fibers for $m \geq 3$ (cf. Theorem 2.8), we are done.

Therefore it remains to verify the Claim.

Let $R_{m}:=H^{0}\left(\omega_{F}^{m}\right)$, we first show that $R_{1} R_{m} \subset R_{m+1}$ has codimension $\leq 1$ for all $m \geq 0$. To see this, we may assume that $F=F_{0}$. Let $Y \in\left|K_{F}\right|$ be a general curve. We have that $Y$ is non-singular, for otherwise $Y$ is singular at the base point of $\left|K_{F}\right|$ and hence $1=Y^{\prime} \cdot Y \geq 2$ for $Y^{\prime} \neq Y \in\left|K_{F}\right|$.

Let $\sigma_{1} \in R_{1}$ be a section defining $Y$, then we have the short exact sequence

$$
\left.0 \rightarrow \omega_{F}^{m} \stackrel{\sigma_{1}}{\longrightarrow} \omega_{F}^{m+1} \longrightarrow \omega_{F}^{m+1}\right|_{Y} \rightarrow 0 .
$$

By the Kawamata-Viehweg vanishing theorem and since $q(F)=0$, we have an exact sequence on global sections

$$
0 \rightarrow R_{m} \stackrel{\sigma_{1}}{\longrightarrow} R_{m+1} \longrightarrow H^{0}\left(\left.\omega_{F}^{m+1}\right|_{Y}\right) \rightarrow 0,
$$

for $m \geq 0$. By Riemann-Roch, one sees that

$$
h^{0}\left(\left.\omega_{F}^{m}\right|_{Y}\right)= \begin{cases}m & \text { if } m=1,2, \\ m-1 & \text { if } m \geq 3 .\end{cases}
$$

We claim that the cokernel of the homomorphism $H^{0}\left(\omega_{F}^{m}\right) \otimes H^{0}\left(\omega_{F}\right) \rightarrow$ $H^{0}\left(\omega_{F}^{m+1}\right)$ has dimension 1 for $m=1$ and $m \geq 3$ and dimension 0 for $m=2$. Consider in fact the exact sequence:

$$
0 \rightarrow \omega_{F}^{m-1} \rightarrow \omega_{F}^{m} \otimes H^{0}\left(\omega_{F}\right) \rightarrow \omega_{F}^{m+1} \rightarrow \mathbb{C}(z) \rightarrow 0
$$

where $z$ is the unique base point of $\left|K_{F}\right|$. If $m \geq 3$, all higher cohomologies vanish and so we get an exact sequence of global sections. An easy spectral sequence argument shows that the sequence

$$
0 \rightarrow H^{0}\left(\omega_{F}^{m-1}\right) \rightarrow H^{0}\left(\omega_{F}^{m}\right) \otimes H^{0}\left(\omega_{F}\right) \rightarrow H^{0}\left(\omega_{F}^{m+1}\right)
$$

is also exact for $m=1,2$. By an easy dimension count, the above statement follows.

We now consider the multiplication map

$$
\varphi_{m}: a_{*} \omega_{X} \otimes a_{*} \omega_{X}^{m} \rightarrow a_{*} \omega_{X}^{m+1}
$$

By the above claim, one sees that $\varphi_{m}$ has cokernel of generic rank $\leq 1$ for all $m \geq 1$ and it is generically surjective for $m=2$.

We now distinguish the following cases:

1. $E_{1}:=a_{*} \omega_{X}$ is indecomposable.

Since $K_{X}$ is full, so is $E_{1}$. By the semipositivity of $a_{*} \omega_{X}$, we have that $\operatorname{deg}\left(E_{1}\right) \geq 0$. If $\operatorname{deg}\left(E_{1}\right)=0$, then $E_{1} \cong U \otimes P$ for some vector bundle $U$ with maximal splitting $\left(\mathcal{O}_{C}, \mathcal{O}_{C}\right)$ and some $P \in \operatorname{Pic}^{0}(C)$. However, $U \otimes P$ is not full. Thus we have $\operatorname{deg}\left(E_{1}\right)>0$ and hence $\mu\left(E_{1}\right) \geq \frac{1}{r k(E)}=\frac{1}{2}$. According to the ring structure given in [At], one sees that $\nu\left(E_{1} \otimes E_{1}\right) \geq \nu\left(E_{1}\right)+\nu\left(E_{1}\right) \geq 1$ and hence $\nu\left(\operatorname{im} \varphi_{1}\right) \geq 1$ by Lemma 4.8. Moreover, the cokernel of $\varphi_{1}$ is $I T^{0}$ and has rank $\leq 1$, 
so $\nu\left(E_{2}\right) \geq 1$ by Lemma 4.9 . Repeatedly applying this argument, one sees that $\nu\left(E_{m}\right) \geq 1$ for $m \geq 5$.

2. $E_{1}$ is decomposable.

Then it is of the form $L \oplus P$ with $\operatorname{deg}(L) \geq 1, \operatorname{deg}(P) \geq 0$. Note that $\varphi_{1}$ factors through $\operatorname{Sym}^{2}\left(E_{1}\right)=P^{2} \oplus L \otimes P \oplus L^{2}$. Moreover, $\operatorname{im}\left(\varphi_{1}\right)$ has rank $\geq 3$ because $\varphi_{1}$ has cokernel of rank $\leq 1$. It follows that $\operatorname{Sym}^{2}\left(E_{1}\right) \cong \operatorname{im}\left(\varphi_{1}\right)$.

Before we move on, notice that $E_{m}$ is $I T^{0}$ for $m \geq 2$ by Lemma 4.1. 2.a. $E_{2}$ is indecomposable.

By Lemma 4.8, $\mu\left(E_{2}\right) \geq 2$ for there is a non-zero map from $L^{2}$ to $E_{2}$. By considering $\varphi_{2}, \ldots, \varphi_{m-1}$ and Lemma 4.9 , one has that $\nu\left(E_{m}\right) \geq 1$ for $m \geq 5$.

2.b. $E_{2}=\mathcal{E}_{1} \oplus \mathcal{E}_{2}$ with ranks 3,1 respectively.

Then $\mu\left(\mathcal{E}_{1}\right) \geq 1$ for there is a non-zero map from $L$ or $L^{2}$ in to $\operatorname{im}\left(\varphi_{1}\right)$ to $\mathcal{E}_{1}$. Since $E_{2}$ is IT0, so is $\mathcal{E}_{2}$. Thus $\mu\left(\mathcal{E}_{2}\right) \geq 1$ for it is a line bundle of $I T^{0}$. Hence we have $\nu\left(E_{2}\right) \geq 1$. Proceeding as above, it follows that $\nu\left(E_{m}\right) \geq 1$ for $m \geq 5$.

2.c. $E_{2}=\oplus \mathcal{E}_{i}$ factors into 4 bundles of rank 1 .

Since $E_{2}$ is $I T^{0}$, so are the bundles $\mathcal{E}_{i}$. Thus $\mu\left(\mathcal{E}_{i}\right) \geq 1$ and hence $\nu\left(E_{2}\right) \geq 1$. Proceeding as above, it again follows that $\nu\left(E_{m}\right) \geq 1$ for $m \geq 5$.

2.d. $E_{2}$ decomposes into bundles of rank $(2,1,1)$ or $(2,2)$.

Since each component is $I T^{0}$, we have $\nu\left(E_{2}\right) \geq \frac{1}{2}$. We then consider the multiplication map

$$
\psi: a_{*} \omega_{X}^{2} \otimes a_{*} \omega_{X}^{2} \rightarrow a_{*} \omega_{X}^{4} .
$$

One can check that $R_{2} R_{2} \subset R_{4}$ has codimension $\leq 1$ (its image in fact contains the image of $R_{1} R_{1} R_{2}$ and hence it contains the image of $R_{1} R_{3}$ which has codimension at most 1$)$. So we conclude that $\nu\left(E_{2} \otimes E_{2}\right) \geq$ $\nu\left(E_{2}\right)+\nu\left(E_{2}\right) \geq 1$ and hence $\nu\left(E_{4}\right) \geq 1$ by Lemma 4.9. Then, by considering $\varphi_{4}, \cdots, \varphi_{m-1}$, it follows that $\nu\left(E_{m}\right) \geq 1$ for $m \geq 5$. This completes the proof.

\section{REFERENCES}

[At] M. Atiyah, Vector bundles over an elliptic curves, Proc. London Math. Soc. 7(1957), 414-452.

[Bo] E. Bombieri, Canonical models of surfaces of general type. Inst. Hautes Études Sci. Publ. Math. 42 (1973), 171-219.

[CH1] J. A. Chen and C. D. Hacon, Linear series of irregular varieties, Algebraic Geometry in East Asia, Japan, 2002, World Scientific Press.

[CH2] J. A. Chen, C. D. Hacon, On algebraic fiber spaces over varieties of maximal Albanese dimension, Duke Math. Jour. 111, (2002), 159-175.

[CH3] A. J. Chen, C. D. Hacon, Pluricanonical maps of varieties of maximal Albanese dimension, Math. Ann. 320 (2001) 2, 367-380.

[GL1] M. Green, R. Lazarsfeld, Deformation theory, generic vanishing theorems, and some conjectures of Enriques, Catanese and Beauville, Invent. Math. 90(1987), 389-407. 
[GL2] M. Green, R. Lazarsfeld, Higher obstructions to deforming cohomology groups of line bundles, Jour. Amer. Math. Soc. 4(1991), 87-103

[Ha] R. Hartshorne, Ample vector bundles on curves. Nagoya Math. J. Vol. 43 (1971), 73-89.

[HM] C. D. Hacon, J. Mc Kernan, Boundedness of pluricanonical maps of varieties of general type. To appear in Invent. Math.

[Ko1] J. Kollár, Higher direct images of dualizing sheaves I, Ann. Math. 123 (1986), 11-42.

[Ko2] J. Kollár, Higher direct images of dualizing sheaves II, Ann. Math. 124 (1986), 171-202.

[Ko3] J. Kollár, Shafarevich maps and automorphic forms, M. B. Porter Lectures, Princeton Univ. Press, Princeton 1995.

[Mu] S. Mukai, Duality between $D(X)$ and $D(\hat{X})$ with its application to Picard sheaves Nagoya math. J. 81 (1981), 153-175.

[Ta] S. Takayama, Pluricanonical systems on algebraic varieties of general type. To appear in Invent. Math.

[To] G. Todorov, Pluricanonical maps for threefolds. Preprint math.AG/0512346.

$[\mathrm{Tu}] \quad \mathrm{L} . \mathrm{W}$. Tu, Semistable Bundles over an elliptic curve, Adv. in Math 98, 1-26 (1993)

[Ts1] H. Tsuji, Pluricanonical systems of projective varieties of general type II. math.CV $/ 0409318$

[Ts2] H. Tsuji, Pluricanonical systems of projective 3-folds of general type. math.AG/0204096

[V1] E. Viehweg, Weak positivity and the additivity of the Kodaira dimension for certain fiber spaces, Adv. Stud. Pure Math., North Holland, 1 (1983) 329-353

[V2] E. Viehweg, Quasi-projective moduli for Polarized Manifolds, Springer, Ergebnisse der Mathematik und ihrer Grenzgebiete 30 (1995)

Department of Mathematics, National Taiwan University, Taipei 10617, TAIWAN.

National Center for Theoretical Sciences, Taipei Office.

E-mail address: jkchen@math.ntu.edu.tw

Department of Mathematics, University of Utah, 155 South 1400

EAst, JWB 233, SAlt LAKE City, UT 84112-0090, USA

E-mail address: hacon@math.utah.edu 